\title{
(6) OPEN ACCESS \\ ADAPT technique with ACE68 and ACE64 reperfusion catheters in ischemic stroke treatment: results from the PROMISE study
}

For numbered affiliations see end of article.

\section{Correspondence to} Professor Peter Schramm, Department of Neuroradiology, University Medical Center Schleswig-Holstein, Luebeck 23538, Germany; Peter. Schramm@uksh.de

Received 31 May 2018 Revised 2 July 2018 Accepted 5 July 2018 Published Online First 30 July 2018

\section{Check for updates}

(c) Author(s) (or their employer(s)) 2019. Re-use permitted under CC BY-NC. No commercial re-use. See rights and permissions. Published by BMJ.

To cite: Schramm P, Navia $P_{\text {, }}$ Papa R, et al.

J Neurolntervent Surg

2019:11:226-231.

\author{
Peter Schramm, ${ }^{1}$ Pedro Navia, ${ }^{2}$ Rosario Papa ${ }^{3}$ Joaquin Zamarro, ${ }^{4}$ \\ Alejandro Tomasello, ${ }^{5,6}$ Werner Weber, ${ }_{1}^{7}$ Jens Fiehler, ${ }^{8}$ Patrik Michel, ${ }^{9}$ Vitor M Pereira, ${ }^{10}$ \\ Timo Krings, ${ }^{11,12}$ Jan Gralla, ${ }^{13}$ Paola Santalucia, ${ }^{14}$ Laurent Pierot, ${ }^{15}$ T H Lo ${ }^{16}$
}

\begin{abstract}
Background and purpose The recent randomized trials demonstrated the benefit of mechanical thrombectomy in stroke therapy. However, treatment using different strategies is an ongoing area of investigation. The PROMISE study analyzed the safety and effectiveness of the Penumbra System with the ACE68 and ACE64 reperfusion catheters in aspiration thrombectomy of stroke, using A Direct Aspiration First Pass Technique (ADAPT).

Methods PROMISE was a prospective study which enrolled 204 patients with intracranial anterior circulation large vessel occlusion (LVO) ischemic stroke in 20 centers from February 2016 to May 2017. Initial treatment was with the ACE68/ACE64 catheters within 6 hours of symptom onset. Imaging and safety review was performed by an independent Core Laboratory and a Clinical Events Committee. The primary angiographic outcome was revascularization to $\mathrm{mTICl} 2 \mathrm{~b}-3$ at immediate post-procedure and the primary clinical outcome was 90-day modified Rankin Scale (mRS) score $\leq 2$. Safety assessment included device- and procedurerelated serious adverse events (SAEs), symptomatic intracranial hemorrhage (sICH), mortality, and embolization of new territory (ENT).
\end{abstract}

Results Enrolled patients had a median age of 74 (IQR 65-80) years and a median admission NIHSS of 16 (IQR 11-20). The post-procedure $\mathrm{mTICI} 2 \mathrm{~b}-3$ revascularization rate was $93.1 \%$ and the 90 -day mRS $0-2$ rate was $61 \%$. Device- and procedure-related SAEs at 24 hours occurred in $1.5 \%$ and $3.4 \%$, respectively, 90 -day mortality was $7.5 \%$, sICH occurred in $2.9 \%$ while ENT occurred in $1.5 \%$.

Conclusions For frontline therapy of LVO stroke, the ACE68/ACE64 catheters for aspiration thrombectomy were found to be safe and showed similar efficacy to randomized trials using other revascularization techniques.

Clinical Trial Registration : NCT02678169;Preresults.

\section{INTRODUCTION}

Six randomized controlled trials (RCTs) established the role of mechanical thrombectomy in early acute ischemic stroke (AIS): ${ }^{1-6}$ in these trials, most of the devices used were stent retrievers. As a result of position statements recommending endovascular therapy, ${ }^{8-10}$ a significant growth in the treatment of patients with emergent large vessel occlusion (ELVO) has been observed and is expected to continue. ${ }^{11-13}$ An operational definition of ELVO to standardize the description of the therapeutic target for endovascular thrombectomy was proposed. ${ }^{14}$ Recently, several studies have investigated patient outcomes after advances in different devices and techniques, increased physician experience, and streamlined in-hospital procedures for endovascular therapy. ${ }^{15-18}$ The Penumbra System (Penumbra, Inc., Alameda, CA) is a family of mechanical thrombectomy devices specifically designed to remove thrombus through aspiration, receiving $510(\mathrm{k})$ clearance from the US FDA in 2007. ${ }^{19} 20$ THERAPY was an RCT assessing aspiration thrombectomy with IV rt-PA versus IV rt-PA alone. ${ }^{21}$ Further developments included ADAPT (A Direct Aspiration First Pass Technique), a thrombectomy approach wherein aspiration alone is used to remove the occlusion, followed by adjunctive therapies if necessary. ${ }^{22-24}$ In addition, findings from the Penumbra 3D RCT, which randomized patients to either a frontline approach with the $3 \mathrm{D}$ stent retriever with aspiration vs aspiration alone, demonstrated no significant difference between treatment groups in the rates of modified Thrombolysis in Cerebral Infarction (mTICI) 2-3 revascularization, 90-day modified Rankin Scale (mRS) $0-2$, symptomatic intracranial hemorrhage $(\mathrm{sICH})$, procedure or device-related serious adverse events (SAEs), or mortality. ${ }^{25}$

At the time of this study's design, the safety and efficacy of the ADAPT technique using ACE68/64 catheters with the Penumbra System (PS) had not been assessed in a prospective study. The aim of a prospective, multicenter, observational, single-arm European Registry on the ACE reperfusion catheters and the Penumbra System in the treatment of acute ischemic stroke (PROMISE) study was to address this need for real-world data on the safety and effectiveness of the PS with the novel Penumbra reperfusion catheters, ACE68/64, in patients with AIS from anterior circulation LVO, treated with the ADAPT technique. The PROMISE study incorporated an integrated aspiration thrombectomy system which includes a Penumbra aspiration pump indicated for revascularization of AIS patients. 


\section{METHODS}

PROMISE was a prospective, multicenter, single-arm, open-label, observational European study to evaluate the safety and effectiveness of the ACE68/64 reperfusion catheters and the PS in the treatment of AIS (ClinicalTrials.gov Identifier, NCT02678169). Patients presenting within 6 hours from symptom onset with an anterior circulation LVO AIS (within the internal carotid artery (ICA) and internal carotid terminus, middle cerebral artery (MCA) - M1/M2 segments) were eligible. This study was a non-inferiority comparison of 90-day mRS 0-2 rates between the ACE Penumbra System group and the intra-arterial therapy (IAT) group in the MR CLEAN trial. ${ }^{1}$ At the time of study design, MR CLEAN established the benefit of intra-arterial therapy against medical management alone and was the only published RCT for our non-inferiority hypothesis and sample size calculation. The main aim of the study, however, was to assess the safety and effectiveness of the ACE68/64 in patients treated with the ADAPT technique. Every patient was checked for study eligibility and recorded on a site-specific Screening Log.

Treatment consisted of the ADAPT technique with thrombo-aspiration using ACE68 and ACE64 as first intention according to site routine practice. For rescue, any approved revascularization device and/or intra-arterial medication was allowed at the operators' discretion, including non-Penumbra devices. Adjunctive therapy was defined as the use of a stent retriever following a reperfusion catheter: before adjunctive therapy, three passes of the ACE catheter were recommended. At the discretion of the clinicians in charge of the patient or the investigator, the patient was pretreated with intravenous thrombolytics (IV tPA), and/or treated under general or local anesthesia, or conscious sedation. Follow-up visits to assess functional outcome, quality of life, and adverse events took place at 24 hours, 7 to 10 days or discharge, 30-days, and 90-days post-procedure. In Europe, the ACE68 and ACE64 catheters were available in 2016 and 2015, respectively.

All procedures were in accordance with national and local ethical and institutional guidelines and site routine clinical practice. The local Ethics Committees approved the study, and the patients or their representatives provided written informed consent, according to local regulations.

\section{Imaging analysis}

All imaging scans (baseline CT/MRI, pre-procedure/procedure DSA, and 24 hours' scan) were reviewed and adjudicated by an independent Core Imaging Laboratory (Toronto Western Hospital, University of Toronto) for mTICI and for procedural and safety assessments (confirmation of vessel damage and embolization of new territory (ENT), identification, and classification of intracranial hemorrhages).

\section{Patients}

Patient eligibility criteria included age of at least 18 years, presentation with anterior circulation LVO within 6 hours of stroke onset or time last known to be well, occlusion of the internal carotid artery (ICA and ICA terminus) or MCA M1/ M2, National Institutes of Health Stroke Scale (NIHSS) $\geq 2$, and pre-stroke $\mathrm{mRS}$ score $\leq 2$. LVO status was determined mostly by CTA $(85.3 \%)$, with some by MRA (14.7\%). Imaging eligibility criteria were CT Alberta Stroke Program Early CT Score (ASPECTS) $\geq 6$ or MR DWI ASPECTS $\geq 5$, and absence of tandem extracranial occlusion or severe arterial stenosis requiring treatment prior to thrombectomy. Study exclusion criteria can be found in the Online Supplement. Up to 210 patients who met eligibility criteria were to be enrolled in a maximum of 25
European investigational sites, and an exclusion log of screened but not enrolled patients was kept at each site.

\section{Primary and secondary endpoints}

Primary endpoints were angiographic revascularization assessed by digital subtraction angiography of the occluded target vessel to mTICI $2 \mathrm{~b}$ or 3 at immediate post-procedure as adjudicated by an independent Core Laboratory and functional independence (mRS 0-2) at 90 days by mRS certified medical personnel, without blinding to the clinical or radiological patient data. Secondary endpoints were any device- and procedure-related SAEs at 24 hours' and 30 days' post-procedure, all-cause morbidity (defined as mRS 3-5) and mortality at 90 days, occurrence of ENT, occurrence of sICH at 24 hours, and occurrence of vessel damage at the end of the ADAPT procedure. Safety was assessed by collecting adverse events data during all study visits. Hemorrhagic transformation was radiologically classified according to the European Cooperative Acute Stroke Study (ECASS) definitions. sICH was defined according to ECASS-II definition ${ }^{26} 27$ as a four point or greater deterioration in the NIHSS within 24 hours after treatment compared with the pre-procedure NIHSS attributable to imaging evidence of an intracranial hemorrhage as adjudicated by the Core Imaging Laboratory. Safety endpoints were adjudicated for severity and causality by an independent Clinical Events Committee (CEC). All data were monitored during on-site visits.

Secondary efficacy endpoints included good functional neurological recovery defined as a reduction of 10 or more points in the NIHSS or a score of $0-1$ at 7 to 10 days' post-procedure, times to $\mathrm{mTICI} \geq 2 \mathrm{~b}$ revascularization, health economics data, and Quality of Life as assessed by EQ-5D-3L score at 90 days compared with 7 to 10 days.

\section{Statistical analysis}

Baseline clinical and imaging data were summarized using standard descriptive statistics. This included the number of observations, mean, median, SD, minimum and maximum for continuous variables, and counts and percentages for discrete variables. All confidence intervals presented were two-sided. All statistical tests were two-tailed with a significance level of 0.05 . Analyses were performed using SAS software (version 9.4; SAS Institute). ${ }^{28}$

The primary effectiveness analysis was an unadjusted non-inferiority comparison between the ACE68/64 reperfusion catheters Penumbra System group and the IAT group from the MR CLEAN trial. For sample size calculation, it was assumed that $38 \%(76 / 200)$ of the PS patients would experience primary endpoint success for 90-day mRS 0-2 compared with $32.6 \%$ (76/233) of the MR CLEAN IAT group: the estimated difference and $95 \% \mathrm{CI}$ for the difference between groups would therefore be $5.4 \%(-3.7 \%, 14.4 \%)$. As the primary analysis, all efficacy and safety outcome measures were analyzed under the intent-to-treat (ITT) principle.

Prespecified analyses of association between primary and selected secondary endpoints (mTICI 2b-3 post-procedure, 90-day mRS 0-2, device- or procedure-related SAEs at 24 hours and 30 days, 90-day mortality, occurrence of sICH) were conducted, with adjustment for several baseline characteristics (age, baseline NIHSS, occlusion location).

\section{RESULTS}

\section{Baseline patient data}

Between February 2016 and May 2017, the PROMISE study enrolled 204 patients across 20 European centers using ADAPT 
with the Penumbra System and ACE68/64 catheters as frontline treatment. For enrollment, 30 (14.7\%) patients were selected by MRI and $174(85.3 \%)$ by CT. Of the 1433 patients screened and documented in site-specific Screening and Enrollment Logs, 1229 patients $(85.8 \%)$ were screen failures and 204 patients $(14.2 \%)$ were enrolled. The five most common reasons for screen failure were no imaging evidence of LVO of the anterior circulation $(22.1 \%, 271 / 1229)$, followed by unknown onset of stroke symptoms with last proof of good health more than 6 hours before $(10.3 \%, 126 / 1229)$, lack of possibility to obtain informed consent $(10.2 \%, 125 / 1229)$, onset of stroke symptoms $>6$ hours $(8.2 \%, 101 / 1229)$ or angiographic evidence of tandem extracranial occlusion or arterial stenosis proximal to occlusion requiring treatment prior to thrombus removal $(8.2 \%$, $101 / 1229)$. Four patients withdrew consent prior to the 90 -day visit, therefore, 200 patients completed the 90-day visit and were included in the follow-up analysis.

Baseline characteristics are displayed in table 1 . The median age was 74 years, ranging from 27 to 96 years, and almost two-thirds of the patients were women $(61.8 \%)$. Thirty-five percent $(35.3 \%)$ of patients were transferred from other hospitals, 79.9\% of stroke onsets were witnessed, and 20.1\% of patients were last known to be well within 6 hours before inclusion without knowing the exact time of onset. The median time from stroke onset to hospital admission was $97 \mathrm{~min}$ (IQR 58.5154.5). The median baseline NIHSS score was 16 (IQR 11-20) and ranged from 2 to 36 . At admission, the median CT ASPECTS was 9, while the median MRI DWI ASPECTS was 8. Occlusions were located in the ICA/carotid T (21.1\%) and MCA $(78.9 \%$; $60.8 \%$ in M1, $18.1 \%$ in M2) and IV rt-PA was administered in 126 patients $(61.8 \%)$, mainly within a bridging protocol $86.5 \%$ $(109 / 126)$. At baseline, mTICI was 0 in most patients $(91.2 \%$, $186 / 204)$ with mTICI 1 in $8.3 \%(17 / 204)$ of patients.

The ACE68 catheter was utilized as frontline treatment in $65.7 \%$ of patients and the ACE64 in 32.8\%: three patients $(1.5 \%)$ were initially treated with other PS devices (table 1). For cases that required adjunctive therapy, the median number of ACE68/64 passes performed before switching was 2 (IQR 1-3). General anesthesia was performed in $58.3 \%$, conscious sedation in $18.1 \%$, and local anesthesia only in $23.5 \%$ of patients. A stent was placed for proximal stenosis or dissection in $2 \%$ of patients, and balloon angioplasty was used for proximal stenosis in $2 \%$ of patients. In $20.9 \%$ of patients, a stent retriever was used after PS as adjunctive treatment, in $13.4 \%$ of cases at the target vessel, $9 \%$ in distal vasculature, and $1.5 \%$ in both. No balloon guide catheters were reported to be used.

\section{Primary outcomes}

The proportion of patients with Core Laboratory-assessed mTICI 2b-3 was 93.1\% (190/204) after all treatment $(39.2 \%$ mTICI 3, 53.9\% mTICI 2b). After Penumbra System treatment alone, $70.6 \%(144 / 204)$ of patients achieved mTICI 2b-3. Out of all patients achieving mTICI 2b-3 after ACE68/64, 74.4\% (90/121) achieved revascularization after the first ACE68/64 pass. At 90 days, $61 \%$ of patients (122/200) achieved good functional outcome (mRS $0-2)$ (table 2 ). The study met its primary effectiveness endpoint (Online Supplement).

\section{Secondary outcomes}

Regarding safety endpoints, the all-cause mortality and morbidity rates at 90 days were $7.5 \%(15 / 200)$ and $31.5 \%(63 / 200)$, respectively, and the 24-hours sICH rate was 2.9\% (6/204). According to MedDRA preferred terms, three patients died due to progression of stroke, two patients died due to cerebrovascular accident,
Table 1 Baseline and procedural characteristics

\begin{tabular}{|c|c|}
\hline Characteristic & Patients (N) \\
\hline Age, years & $\begin{array}{l}71.3 \pm 12.4(204)(\text { mean } \pm S D) \\
74 / 65-80 \text { (median/lQR) }\end{array}$ \\
\hline Female & $61.8 \%(126 / 204)$ \\
\hline Baseline NIHSS & $\begin{array}{l}15.4 \pm 5.8(204)(\text { mean } \pm S D) \\
16 / 11-20(\text { median/lQR) }\end{array}$ \\
\hline$<16$ & $46.6 \%(95 / 204)$ \\
\hline $16-20$ & $32.8 \%(67 / 204)$ \\
\hline$>20$ & $20.6 \%(42 / 204)$ \\
\hline CT ASPECTS*: per site assessment & $\begin{array}{l}8.7 \pm 1.2(173)(\operatorname{mean} \pm S D) \\
9 / 8-10 \text { (median/IQR) }\end{array}$ \\
\hline MR DWI ASPECTS: per site assessment & $\begin{array}{l}7.4 \pm 1.5(30)(\text { mean } \pm S D) \\
8 / 6-8(\text { median/IQR })\end{array}$ \\
\hline IV rt-PA administered prior to procedure & $61.8 \%(126 / 204)$ \\
\hline $\begin{array}{l}\text { Time from symptom onset to hospital admission } \\
\text { (min) }\end{array}$ & $\begin{array}{l}115.3 \pm 78.6(204)(\text { mean } \pm S D) \\
97.0 / 58.5-154.5(\text { median/IQR) }\end{array}$ \\
\hline \multicolumn{2}{|l|}{ Site of vessel occlusion } \\
\hline Internal carotid artery/carotid T & $21.1 \%(43 / 204)$ \\
\hline Middle cerebral artery & $78.9 \%(161 / 204)$ \\
\hline First segment (M1) & $60.8 \%(124 / 204)$ \\
\hline Second segment (M2) & $18.1 \%(37 / 204)$ \\
\hline
\end{tabular}

\section{Procedural information}

Penumbra ACE Catheters, $(\% n / N) \quad$ All patients $(n=204)$

Frontline device

$\begin{array}{ll}\text { ACE68 } & 65.7 \%(134 / 204) \\ \text { ACE64 } & 32.8 \%(67 / 204)\end{array}$

Other Penumbra catheters: 3MAX, 5MAX, ACE $60 \quad 1.5 \%(3 / 204) \dagger$

Frontline ACE68/64 catheter passes $\quad 1.6 \pm 0.9(201)($ mean $\pm S D(N))$ $1 / 1-2$ (median/IQR)

Additional mechanical intervention $(\% n / N) \ddagger \quad$ ACE 68/64 PATIENTS $n=201$

Stent retriever after Penumbra System, total $20.9 \%(42 / 201)$

Target vessel/remaining clot $13.4 \%(27 / 201)$

Distal vasculature $9 \%(18 / 201)$

Stent for proximal stenosis or dissection $\quad 2 \%(4 / 201)$

Balloon angioplasty for proximal stenosis $\quad 2 \%(4 / 201)$

Catheter from other manufacturer $1 \%(2 / 201)$

Data are $\%(\mathrm{n} / \mathrm{N})$ or mean $\pm \mathrm{SD}(\mathrm{N})$ (median) (IQR). ASPECTS, Alberta Stroke Program Early CT Score; IQR, interquartile range; NIHSS, National Institutes of Health Stroke Scale; mRS, modified Rankin Scale.

*One patient with ASPECTS score not available due to a previous stroke. Principal Investigator confirmed the inclusion of the patient.

tThree patients were treated with ACE60, 3MAX, or 5MAX, admitted under protocol version $B$.

‡Patients may have more than one additional mechanical intervention.

and the remaining 10 patients died due to different causes (cerebral hemorrhage, hemorrhagic transformation stroke, ischemic stroke, seven others not related to stroke). The ENT rate was $1.5 \%(3 / 204)$, vessel perforation rate was $0.5 \%(1 / 204)$, distal emboli rate was $1.0 \%(2 / 204)$, and vessel dissection rate was $2.5 \%(5 / 204)$ (table 2$)$. The frequency of new ischemic stroke within 24 hours was $0 \%$.

Per adjudication by the CEC, there were nine device- and procedure-related SAEs (4.4\%) at 30 days, of which seven $(3.4 \%)$ occurred within 24 hours. These events included carotid artery dissection $(n=2)$, cerebral artery embolism $(n=2)$, cerebral artery occlusion $(n=1)$, cerebral hematoma $(n=1)$, hemorrhagic 


\begin{tabular}{|c|c|}
\hline Outcome & $\%(n / N)$ \\
\hline \multicolumn{2}{|l|}{ Primary endpoints } \\
\hline $\begin{array}{l}\mathrm{mTICI} 2 \mathrm{~b}-3 \text { at post-procedure: per core } \\
\text { laboratory* }\end{array}$ & $93.1 \%(190 / 204)$ \\
\hline mRS $0-2$ at 90 days $\dagger$ & $61 \%(122 / 200)$ \\
\hline \multicolumn{2}{|l|}{ Secondary endpoints } \\
\hline Neurologic improvement‡ & $67.9 \%(127 / 187)$ \\
\hline Device-related SAEs at 24 hours: per CEC & $1.5 \%(3 / 204)$ \\
\hline Device-related SAEs at 30 days: per CEC & $2.0 \%(4 / 204)$ \\
\hline Procedure-related SAEs at 24 hours: per CEC & $3.4 \%(7 / 204)$ \\
\hline Procedure-related SAEs at 30 days: per CEC & $4.4 \%(9 / 204)$ \\
\hline All-cause mortality at 90 days§ & $7.5 \%(15 / 200)$ \\
\hline Morbidity at 90 days $\|$ & $31.5 \%(63 / 200)$ \\
\hline $\begin{array}{l}\text { Symptomatic intracranial hemorrhage at } \\
24 \text { hours: per CEC\# }\end{array}$ & $2.9 \%(6 / 204)$ \\
\hline Embolization of new territory: per CEC & $1.5 \%(3 / 204)$ \\
\hline Vessel damage: per CEC & $2.9 \%(6 / 204)$ \\
\hline Vessel perforation & $0.5 \%(1 / 204)$ \\
\hline Vessel dissection & $2.5 \%(5 / 204)$ \\
\hline Symptom onset to ASPECTS CT/MRI, minutes & $\begin{array}{l}137.9 \pm 71.0(204)(\text { mean } \pm \text { SD) } \\
127.5 / 81.5-188.0(\text { median/IQR })\end{array}$ \\
\hline Imaging to arterial puncture, minutes & $\begin{array}{l}72.2 \pm 44.2(204)(\text { mean } \pm S D) \\
61.5 / 41.0-91.5 \text { (median/lQR) }\end{array}$ \\
\hline Arterial puncture to revascularization, minutes & $\begin{array}{l}40.1 \pm 27.2(204)(\text { mean } \pm S D) \\
31.0 / 20.0-53.0 \text { (median/lQR) }\end{array}$ \\
\hline $\begin{array}{l}\text { EQ-5D-3L VAS improvement at } 90 \text { days } \\
\text { compared with } 7-10 \text { days }\end{array}$ & $\begin{array}{l}4.7 \pm 20.4(119)(\text { mean } \pm S D) \\
5 / 0-15(\text { median/lQR) }\end{array}$ \\
\hline 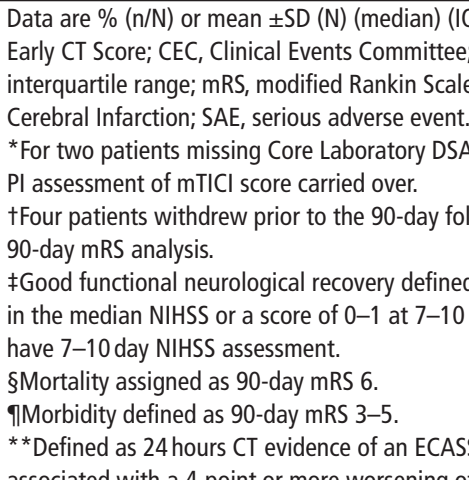 & $\begin{array}{l}\text { R). ASPECTS, Alberta Stroke Program } \\
\mathrm{Cl} \text {, confidence interval; IQR, } \\
\mathrm{mTICI} \text {, modified Thrombolysis in } \\
\text { review due to vessel not visualized, } \\
\text { ow-up and were excluded from } \\
\text { as a reduction of } 10 \text { or more points } \\
\text { days. Seventeen patients did not }\end{array}$ \\
\hline
\end{tabular}

cerebral infarction $(n=1)$, subarachnoid hemorrhage $(n=1)$, and vascular pseudoaneurysm $(n=1)$. Regarding vessel damage events (serious and non-serious), there were five arterial dissections, all of which were extracranial, and one perforation during stent retriever use in distal vasculature. Four of the five dissections were unrelated to ACE catheter use and possibly/probably/ definitely related to the procedure.

Regarding secondary efficacy endpoints, good functional neurological recovery was achieved in $67.9 \%$ of patients (127/187), with $35.3 \%(66 / 187)$ having NIHSS 0-1. The times to revascularization endpoint data are shown in table 2 . The median reperfusion time (arterial access to mTICI $2 \mathrm{~b}-3$ or final angiogram if $2 \mathrm{~b}-3$ was not achieved) was $31 \mathrm{~min}$ (IQR 20-53), and the median time from stroke onset to mTICI $2 \mathrm{~b}-3$ or final angiogram was $245.5 \mathrm{~min}$ (IQR 192-305). The current time metrics are shown in figure 1. Please see Online Supplement for
A

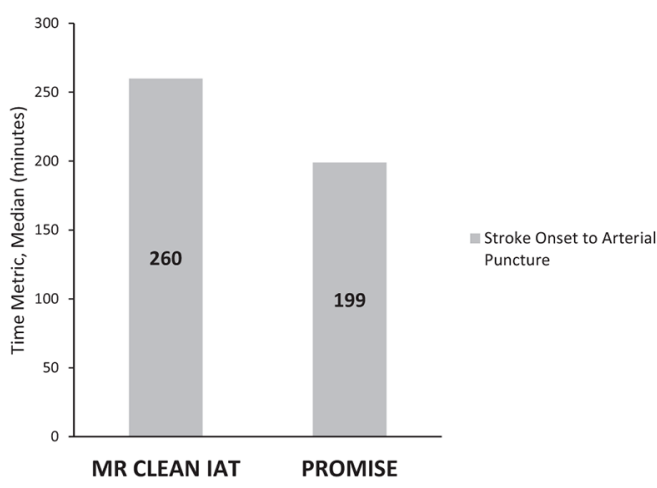

B
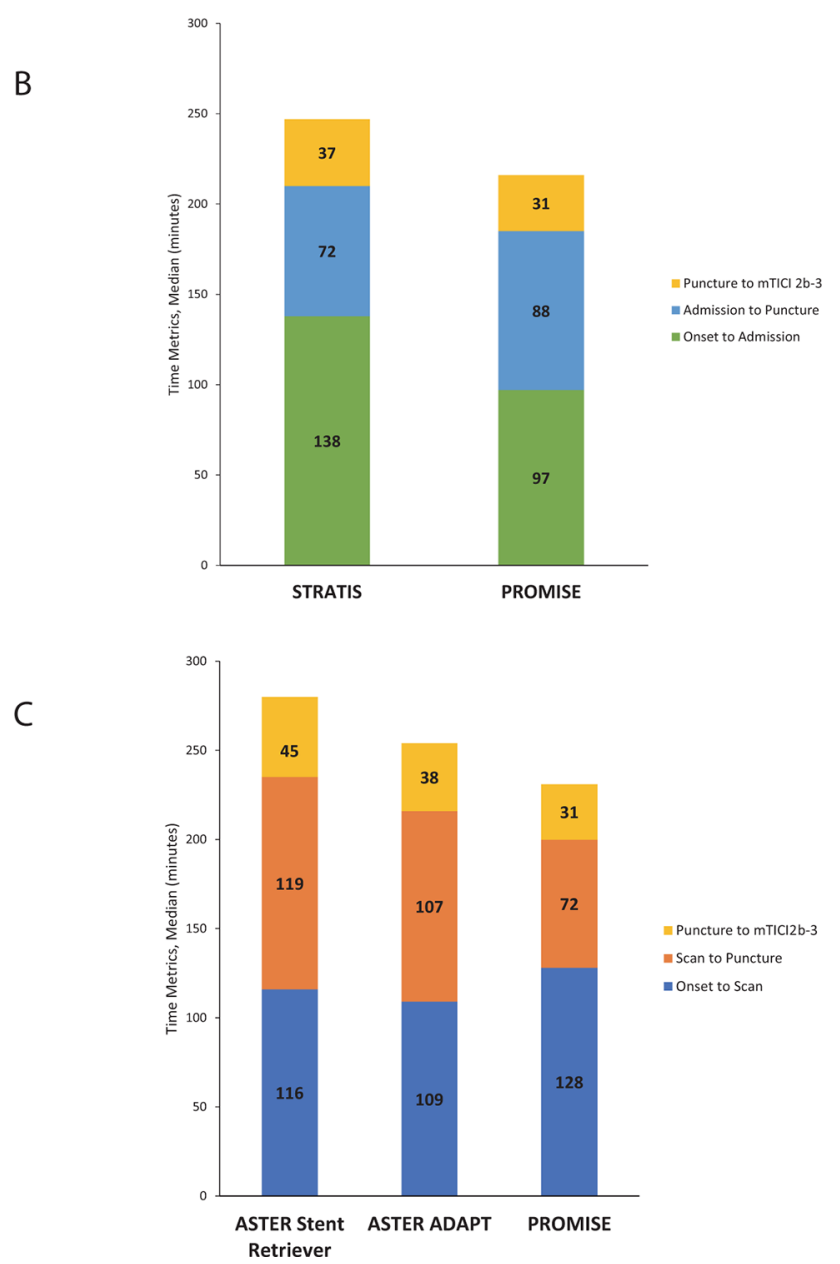

Figure 1 Comparison of PROMISE time metrics with MR CLEAN, STRATIS, and ASTER trials (A) MR CLEAN and PROMISE procedural time comparison. (B) STRATIS and PROMISE procedural time comparison. (C) ASTER and PROMISE procedural time comparison.

details of safety events, primary effectiveness analysis, and additional process times.

Self-reported assessment on quality of life at 90 days compared with 7 to 10 days included improvement in mobility (9.9\%), self-care (14.5\%), usual activities (19.0\%), and pain/discomfort (7.1\%), and worsening in anxiety/depression (4.2\%). Median duration of index hospitalization was 8 days (IQR 5-11), which is typical per European standard of care, and all-cause rehospitalization rate from discharge to 90 days was $8.3 \%$ (17/204). Of note, $73.5 \%$ of patients $(150 / 204)$ were back home by 90 days. 


\section{Subgroup analyses}

In pre-specified subgroup analyses of primary and selected secondary endpoints, several baseline covariates were examined including stroke severity per NIHSS $(<16,16-20$, or $>20)$, age $(<80$ or $\geq 80$ years), and occlusion location. In unadjusted analysis, lower baseline NIHSS and younger age ( $<80$ years) were predictors of functional independence. In addition, younger age ( $<80$ years) was a predictor of decreased mortality. No impact of higher baseline NIHSS on the occurrence of sICH or mortality was observed. In analysis adjusted for the significant predictors of baseline or outcome differences, there were no differences in rates of 90-day mRS 0-2, mTICI 2b-3, or mortality between transfer and direct-admit patients. For subgroup analysis details, please see Online Supplement.

\section{DISCUSSION}

Results from the PROMISE study supported the safety and effectiveness of the ACE68 and ACE64 reperfusion catheters with the PS in patients with AIS secondary to anterior LVO, using the ADAPT technique as frontline therapy in real-world practice. $\mathrm{mTICI} 2 \mathrm{~b}-3$ post-procedure was achieved in $93.1 \%$ of cases (including $20.9 \%$ of patients who were also treated with stent-retrievers), and $61 \%$ of cases achieved 90-day mRS $0-2$. The median time from arterial puncture to mTICI $2 \mathrm{~b}-3$ was $31 \mathrm{~min}$. The safety profile was favorable with $7.5 \%$ early mortality, $2.9 \%$ sICH, and $1.5 \%$ ENT rates.

In the ASTER RCT which randomly assigned patients to a frontline approach with contact aspiration or stent retriever, therapy with the ADAPT technique was not superior to stent retrievers in safety or efficacy. ${ }^{16} \mathrm{~A}$ recent meta-analysis (HERMES) pooled patient-level data from five RCTs comparing endovascular thrombectomy to standard medical care in patients with AIS. It showed the benefit of mechanical thrombectomy devices over medical management alone. ${ }^{7}$ The STRATIS study determined that outcomes observed in a meta-analysis (SEER Collaboration) pooling patient-level data from four RCTs could be reproduced in a large real-world cohort. ${ }^{15}$

Data from the current study were compared with published literature. Compared with ASTER, mTICI 2b-3 was achieved in 93.1\% of patients in PROMISE (20.9\% stent retriever use) vs $85.4 \%$ in ASTER (32.8\% stent retriever use). Compared with the endovascular treatment groups in HERMES, STRATIS, and ASTER, the proportion of patients with 90-day mRS scores 0-2 was higher in PROMISE (61\% vs 46\% in HERMES, $56.5 \%$ in STRATIS, and $45.3 \%$ in ASTER aspiration). Age and baseline NIHSS in these trials were comparable to the PROMISE population, although other characteristics, such as tandem occlusion, may vary. The 90-day mortality rate was satisfactory in PROMISE (7.5\% vs $15.3 \%$ in HERMES, $14.4 \%$ in STRATIS, $19.3 \%$ in ASTER aspiration). The rate of sICH in PROMISE was lower than HERMES and ASTER and higher than STRATIS $(2.9 \%$ vs 4.4\% in HERMES, $1.4 \%$ in STRATIS, 5.3\% in ASTER aspiration). The rate of ENT observed in PROMISE was lower than ASTER and higher than STRATIS (1.5\% vs $0.8 \%$ in STRATIS, $3.7 \%$ in ASTER aspiration). It should be noted that ASTER used almost exclusively ACE64 and 5MAX catheters over ACE68 (65 ACE64, 1 ACE68, 63 5MAX devices were used frontline in the aspiration group).

The improvement in revascularization rates observed in PROMISE might be due to the introduction of the ACE68/64 catheters, along with improved technical skills due to the higher volume of endovascular treatment in recent years. On the other hand, improvements of in-hospital processing times implemented after the introduction of new stroke treatment guidelines $^{8-10}$ as seen in the rapid symptom onset to arterial puncture time (comparison with MR CLEAN, STRATIS, and ASTER in figure 1) might also be correlated with better patient outcomes observed in PROMISE. These observations suggest that the ADAPT technique is an effective method, and that the rate of complications, especially of sICH, is low. This favorable safety profile may arise from the use of the recently introduced large-bore catheters with improved trackability, resulting in less manipulation with multiple devices. Though not a prespecified analysis, no significant differences in safety or effectiveness outcomes were noted between the two catheter groups, ACE64 or ACE68. There were no differences in baseline patient characteristics, except for more cardiac heart failure, less peripheral artery disease, and higher pre-stroke mRS $>0$, in the ACE68 group. The first-pass success rate with ADAPT was $60.2 \%$, with no significant difference between catheter sizes $(58.2 \%$ vs $61.2 \%$, ACE64 vs ACE68). Outcomes should be evaluated with caution due to the unknown impact of the baseline covariates. The potential impact of catheter size remains to be elucidated in a larger study with a matched patient population. In the PROMISE study, balloon guide catheters were not used due to lesional aspiration at the proximal face of the clot.

The primary limitation of the PROMISE study was inherent in its study design and lack of a randomized controlled comparison, and the absence of blinding of the 3-month mRS assessment. Although PROMISE study criteria excluded patients with tandem lesions requiring treatment prior to aspiration, nine patients $(4.4 \%)$ with proximal stenosis were enrolled and five required intervention (two stenting and angioplasty, one stenting, and two angioplasty alone), which may be a lower proportion compared with other studies. The major strengths of this study include evaluation by an independent Core Laboratory and a Clinical Events Committee, prospective and consecutive enrollment of patients to minimize potential bias associated with a registry, all data being monitored during on-site visits, and data collected from real-world clinical practice across Europe.

\section{SUMMARY}

As technical and clinical developments in stroke treatment evolve, these results suggest that in current practice, the ADAPT technique with ACE68/ACE64 offers equal or better results compared with other techniques. The findings from the PROMISE study support the use of aspiration with ACE68/ACE64 reperfusion catheters as a frontline therapy in the treatment of patients with ischemic stroke from large vessel occlusion.

\section{Author affiliations}

'Department of Neuroradiology, University Medical Center Schleswig-Holstein, Luebeck, Germany

${ }^{2}$ Radiology-Interventional Neuroradiology, Hospital Universitario Donostia, San Sebastian, Spain

${ }^{3}$ Radiology, Universita degli Studi di Messina, Messina, Italy

${ }^{4}$ Interventional Neuroradiology, Hospital Universitario Virgen de la Arrixaca, Murcia, Spain

${ }^{5}$ Department of Radiology, Section of Interventional Neuroradiology, Barcelona, Spain

${ }^{6}$ Vall d'Hebron Research Institute (VHIR), Barcelona, Spain

${ }^{7}$ Radiology and Neuroradiology, Ruhr-University Bochum, University Medical Center Langendreer, Bochum, Germany

${ }^{8}$ Department of Neuroradiology, University Medical Center Hamburg Eppendorf, Hamburg, Germany

${ }^{9}$ University of Lausanne, Lausanne, Switzerland

${ }^{10}$ Medical Imaging, Toronto Western Hospital, Toronto, Ontario, Canada

${ }^{11}$ Division of Neuroradiology, Department of Medical Imaging, Toronto Western

Hospital, University Health Network, Toronto, Ontario, Canada

${ }^{12}$ Division of Neurosurgery, Department of Surgery, Toronto Western Hospital,

University Health Network, Toronto, Ontario, Canada

${ }^{13}$ Department for Diagnostic and Interventional Neuroradiology, Inselspital, University 
of Bern, Bern, Switzerland

${ }^{14}$ IRCCS Centro Neurolesi Bonino Pulejo, Piemonte Hospital, Messina, Italy

${ }^{15}$ Radiology, University Hospital Reims, Reims, France

${ }^{16}$ Department of Interventional Radiology and Interventional Neuroradiology,

Universitair Medisch Centrum Utrecht, Utrecht, The Netherlands

Contributors All authors made a substantial, direct, and intellectual contribution to the work.

Funding This study was funded by Penumbra, Inc (9508).

Competing interests The following investigators report financial conflicts with this study: PS reports being a consultant for Penumbra and Stryker, and receiving research support from Penumbra, Philips, and Siemens. RP reports proctoring and consulting for Medtronic, Penumbra, and Implemed. WW reports a proctor agreement with Microvention/Terumo, Medtronic, and Phenox, and being a consultant with Penumbra (speaker and chairman fees). JF reports being a consultant for Penumbra, Acandis, Boehringer Ingelheim, Cerenovus, Covidien, Medtronic, Microvention, Route92, and Stryker, and receiving research support from the German Ministry of Science and Education (BMBF), German Ministry of Economy and Innovation (BMWi), German Research Foundation (DFG), European Union (EU), Hamburgische Investitions- und Förderbank (IFB), Medtronic, Microvention, Philips, and Stryker. PM reports speaker and consulting fees from Medtronic, used for stroke education. TK and VMP received funding from the sponsor for imaging core lab oratory activities. VMP reports being a consultant for Penumbra (PROMISE study), Medtronic, Stryker, and Neurovasc. LP received funding from Penumbra for CEC activities and is a consultant for Balt, Cerenovus/Neuravi, and Microvention.

Patient consent Not required.

Ethics approval The local Ethics Committees approved the study, and the patients or their representatives provided written informed consent, according to local regulations

Provenance and peer review Not commissioned; externally peer reviewed.

Data sharing statement Because of the sensitive nature of the data collected for this study, requests to access the dataset from qualified researchers trained in human subject confidentiality protocols may be sent to Penumbra, Inc. at promise@ penumbrainc.com.

Open access This is an open access article distributed in accordance with the Creative Commons Attribution Non Commercial (CC BY-NC 4.0) license, which permits others to distribute, remix, adapt, build upon this work non-commercially, and license their derivative works on different terms, provided the original work is properly cited, appropriate credit is given, any changes made indicated, and the use is non-commercial. See: http://creativecommons.org/licenses/by-nc/4.0/.

\section{REFERENCES}

1 Berkhemer OA, Fransen PS, Beumer D, et al. A randomized trial of intraarterial treatment for acute ischemic stroke. N Engl J Med 2015:372:11-20.

2 Goyal M, Demchuk AM, Menon BK, et al. Randomized assessment of rapid endovascular treatment of ischemic stroke. N Engl J Med 2015;372:1019-30.

3 Campbell BC, Mitchell PJ, Kleinig TJ, et al. Endovascular therapy for ischemic stroke with perfusion-imaging selection. N Engl J Med 2015:372:1009-18.

4 Saver JL, Goyal M, Bonafe A, et al. Stent-retriever thrombectomy after intravenous t-PA vs. t-PA alone in stroke. N Engl J Med 2015;372:2285-95.

5 Jovin TG, Chamorro A, Cobo E, et al. Thrombectomy within 8 hours after symptom onset in ischemic stroke. N Engl J Med 2015;372:2296-306

6 Bracard S, Ducrocq X, Mas JL, et al. Mechanical thrombectomy after intravenous alteplase versus alteplase alone after stroke (THRACE): a randomised controlled trial. Lancet Neurol 2016:15:1138-47.

7 Goyal M, Menon BK, van Zwam WH, et al. Endovascular thrombectomy after large-vessel ischaemic stroke: a meta-analysis of individual patient data from five randomised trials. Lancet 2016;387:1723-31.
8 Mocco J, Fiorella D, Fargen KM, et al. Endovascular therapy for acute ischemic stroke is indicated and evidence based: a position statement. J Neurointerv Surg 2015;7:79-81.

9 Powers WJ, Derdeyn CP, Biller J, et al. 2015 American Heart Association/American Stroke Association Focused update of the 2013 guidelines for the arly management of patients with acute ischemic stroke regarding endovascular treatment: guideline for healthcare professionals rom the American Heart Association/American Stroke Association. Stroke 2015;46:3020-35.

10 Wahlgren N, Moreira T, Michel P, et al. Mechanical thrombectomy in acute ischemic stroke: consensus statement by ESO-Karolinska Stroke Update 2014/2015, supported by ESO, ESMINT, ESNR and EAN. Int J Stroke 2016;11:134-47.

11 Hirsch JA, Yoo AJ, Nogueira RG, et al. Case volumes of intra-arterial and intravenous treatment of ischemic stroke in the USA. J Neurointerv Surg 2009;1:27-31.

12 Rai AT, Seldon AE, Boo S, et al. A population-based incidence of acute large vessel occlusions and thrombectomy eligible patients indicates significant potential for growth of endovascular stroke therapy in the USA. J Neurointerv Surg 2017:9:722-6.

13 Williams MM, Wilson TA, Leslie-Mazwi T, et al. The burden of neurothrombectomy call: a multicenter prospective study. J Neurointerv Surg 2018. doi: 10.1136/ neurintsurg-2018-013772. [Epub ahead of print 20 Apr 2018].

14 Leslie-Mazwi T, Chandra RV, Baxter BW, et al. ELVO: an operational definition. J Neurointerv Surg 2018;10:507-9.

15 Mueller-Kronast NH, Zaidat 00, Froehler MT, et al. Systematic evaluation of patients treated with neurothrombectomy devices for acute ischemic stroke: primary results of the Stratis Registry. Stroke 2017;48:2760-8.

16 Lapergue B, Blanc R, Gory B, et al. Effect of endovascular contact aspiration vs stent retriever on revascularization in patients with acute ischemic stroke and large vessel occlusion: the Aster randomized clinical trial. JAMA 2017:318:443-52.

17 Frei D, McGraw C, McCarthy K, et al. A standardized neurointerventional thrombectomy protocol leads to faster recanalization times. J Neurointerv Surg 2017:9:1035-40.

18 Menon BK, Sajobi TT, Zhang Y, et al. Analysis of workflow and time to treatment on thrombectomy outcome in the Endovascular Treatment for Small Core and Proximal Occlusion Ischemic Stroke (ESCAPE) randomized, controlled trial. Circulation 2016:133:2279-86.

19 Penumbra Pivotal Stroke Trial Investigators. The Penumbra pivotal stroke trial: safety and effectiveness of a new generation of mechanical devices for clot removal in intracranial large vessel occlusive disease. Stroke 2009;40:2761-8.

20 Tarr R, Hsu D, Kulcsar Z, et al. The POST trial: initial post-market experience of the Penumbra system: revascularization of large vessel occlusion in acute ischemic stroke in the United States and Europe. J Neurointerv Surg 2010;2:341-4.

21 Mocco J, Zaidat 00, von Kummer R, et al. Aspiration thrombectomy after intravenous alteplase versus intravenous alteplase alone. Stroke 2016:47:2331-8.

22 Kowoll A, Weber A, Mpotsaris A, et al. Direct aspiration first pass technique for the treatment of acute ischemic stroke: initial experience at a European stroke center. J Neurointerv Surg 2016:8:230-4.

23 Lapergue B, Blanc R, Guedin P, et al. A Direct Aspiration, First Pass Technique (ADAPT) versus stent retrievers for acute stroke therapy: an observational comparative study. AJNR Am J Neuroradiol 2016;37:1860-5.

24 Turk AS, Frei D, Fiorella D, et al. ADAPT FAST study: a direct aspiration first pass technique for acute stroke thrombectomy. J Neurointerv Surg 2014;6:260-4.

25 Nogueira RG, Frei D, Kirmani JF, et al. Safety and efficacy of a 3-dimensional stent retriever with aspiration-based thrombectomy vs aspiration-based thrombectomy alone in acute ischemic stroke intervention: a randomized clinical trial. JAMA Neurol 2018;75:304-31.

26 Trouillas P, von Kummer R. Classification and pathogenesis of cerebral hemorrhages after thrombolysis in ischemic stroke. Stroke 2006;37:556-61.

27 Hacke W, Kaste M, Fieschi C, et al. Randomised double-blind placebo-controlled trial of thrombolytic therapy with intravenous alteplase in acute ischaemic stroke (ECASS II). Second European-Australasian Acute Stroke Study Investigators. Lancet 1998:352:1245-51

28 SAS Institute, Inc. SAS/STAT 9.3 User's Guide. Cary, NC: SAS Institute, 2011. 